\title{
TESTOSTERONE REPLACEMENT THERAPY AND CARDIOVASCULAR RISK
}

\author{
S.V. Berdnikov' ${ }^{1}$ O.A. Berdnikova ${ }^{2}$ \\ ${ }^{1}$ European medical center, Moscow 129090, Russian Federation \\ ${ }^{2}$ Oryol Regional Hospital, Oryol 302028, Russian Federation
}

\begin{abstract}
Testosterone deficiency is a common problem affecting the quality of life of men. In cohort studies, a direct link between low levels of endogenous testosterone and coronary heart disease, diabetes mellitus, insulin resistance has been identified. A large number of observational researches had been conducted which studied the efficacy and safety of testosterone treatment, indicated contradictory results. At the same time, the number of patients receiving testosterone replacement therapy is steadily increasing. In this review, current data on the cardiovascular safety of testosterone replacement therapy are considered.

Key words: testosterone deficiency, testosterone replacement therapy, cardiovascular risk.

Citation: Berdnikov SV, Berdnikova OA. Testosterone replacement therapy and cardiovascular risk. Siberian Medical Review. 2017;(2): 14-21. DOI: $10.20333 / 2500136-2017-2-14-21$
\end{abstract}

Современные демографические тенденции предполагают прогрессивное старение населения. В 2016 году Росстат опубликовал прогноз ожидаемой продолжительности жизни в РФ к 2030 году, который имеет пессимистичный и оптимистичный сценарии, а именно 73,2 и 77,3 года, соответственно [27]. Логично предположить, что распространенность хронических заболеваний, коморбидность пациентов будут постепенно увеличиваться. В этой связи, важнейшей задачей врача становится не только выбор стратегий снижающих общую смертность, но и улучшающих качество жизни возрастных пациентов.

Среди проблем, значительно влияющих на качество жизни пациентов можно назвать дефицит половых гормонов. Аефицит половых гормонов в менопаузе у женщин и дефицит тестостерона у мужчин вызывают схожие состояния: сексуальную дисфункцию, снижение полового влечения, остеопороз, увеличение массы жировой ткани, депрессию [40]. Эстрогенпрогестиновая заместительная терапия у женщин достаточно хорошо изучена. В настоящее время ее вклад в повышение кардиоваскулярной смертности общепризнан и не вызывает сомнения [8]. Показания к эстроген-прогестиновой заместительной терапии у женщин в менопаузе сужены, при этом основной акцент при ее проведении делается на регулярном скрининге кардиоваскулярных факторов риска и своевременном прекращении терапии при повышении риска сердечно-сосудистых катастроф.

В последние годы фокус сместился с гормонзаместительной терапии у женщин на тестостерон-заместительную терапию (ТЗТ) у мужчин при тестостерон-дефицитных состояниях. Проведено большое количество обсервационных исследований, изучающих возможные преимущества лечения тестостероном, которые показали достаточно противоречивые результаты. Вместе с тем, количество пациентов, получающих ТЗТ неуклонно увеличивается. Более того, в практике кардиолога увеличивается доля больных, которые получили рекомендации отменить гиполипидемические препараты (статины) из-за их способности подавлять выработку тестостерона. В этой связи обзор современных научных данных, касающихся кардиологической безопасности ТЗТ, представляется весьма своевременным.

Изменение соgержания тестостерона в крови с возрастом

Многочисленные исследования демонстрируют снижение с возрастом сывороточных концентраций свободного тестостерона с параллельным увеличением содержания полового гормон-связывающего глобулина (SHBG) [22]. Тем не менее, скорость снижения концентрации тестостерона относительно невелика. Так в исследовании EMAS, включившем 3220 мужчин в возрасте от 40 до 79 лет, концентрация общего и свободного тестостерона в сыворотке крови снижалась на $0,4 \%$ и 1,3\% в год, соответственно [12, 42]. Сывороточные концентрации свободного тестостерона снижаются с увеличением возраста в большей степени, по сравнению с общим тестостероном: так в Massachusetts Male Aging Study сывороточная концентрация свободного тестостерона снижалась на 2,8\% в год [14]. Современное определение позднего мужского гипогонадизма включает, по крайней мере, три клинических симптома в дополнение к документированному снижению уровня тестостерона ниже 11 нмоль/л: 1) потерю утренней эрекции, 2) снижение полового влечения и 3) эректильную дисфункцию. Используя это определение, распространенность гипогонадизма увеличивается с 0,1\% у мужчин в возрасте 40-49 лет до 5,1\% у мужчин в возрасте 70-79 лет [42].

Влияние эндогенного тестостерона на серgечно-сосуguстую систему

Влияние тестостерона на сердечно-сосудистую систему в экспериментах на животных показывает противоречивые результаты. В исследовании Liu et al. размер ишемизированного миокарда крыс при моделировании инфаркта был меньшим у животных с нормальным уровнем свободного тестостерона, по сравнению с животными, которым выполнили орхиэктомию и не проводили гормон-заместительную терапию [32]. Аанный эффект ТЗТ в последствии был неоднократно подтвержден $[31,33,41]$. Целый ряд исследований демонстрирует способность тестостерона вызывать вазодилатацию [1], снижать концентрацию глюкозы крови $[3,29,39]$ и массу жировой ткани $[7,12,23]$. В то же время, были выявлены нежелательные эффекты тестостерона, опосредованные вазоконстрикцией $[2,10]$, активацией путей передачи сигналов смерти на клеточном уровне [13], а также воспалением $[16,28]$ (табл.).

Исследования, описывающие взаимосвязь между тестостероном и атеросклерозом вызвали оживленную полемику в научной печати. Многие исследования показывают, что тестостерон подавляет развитие атеросклероза $[1,21,35,47]$. В то же время другие авторы демонстрируют негативный эффект тестостерона на скорость атеросклеротических изменений сосудистой стенки $[11,34,36,46]$. Papamitsou et al. впервые продемонстрировали прямое повреждающее действие супрафизиологических концентраций тестостерона на миокард крыс, которое проявлялось в гипертрофии и 
Эффекты тестостерона

\begin{tabular}{|c|c|}
\hline Положительные эффекты & Отрицательные эффекты \\
\hline $\begin{array}{l}\text { Антиаритмический эффект: } \\
\text { 1. Более короткий потенциал действия } \\
\text { 2. Укорочение интервала QТс } \\
\end{array}$ & $\begin{array}{l}\text { Вазоконстрикция: } \\
\text { 1. Спазм артерий в эксперименте } \\
\text { 2. Ослабление вазидилатирующего эффекта аденозина }\end{array}$ \\
\hline $\begin{array}{l}\text { Кардиопротекция: } \\
\text { 1. Уменьшение объема ишемического повреждения миокарда в экспери- } \\
\text { менте } \\
\text { 2. Повышение стабильности митохондрий }\end{array}$ & $\begin{array}{l}\text { Атеросклероз: } \\
\text { 1. Экспрессия проатерогенных генов } \\
\text { 2. Экспрессия молекул адгезии на поверхности эндотелиаль- } \\
\text { ных клеток }\end{array}$ \\
\hline $\begin{array}{l}\text { Вазодилатация: } \\
\text { 1. Ингибирование L-типа кальциевых каналов }\end{array}$ & $\begin{array}{l}\text { Воспаление: } \\
\text { 1. Повышение продукции IL-10 }\end{array}$ \\
\hline $\begin{array}{l}\text { Метаболический синдром: } \\
\text { 1. Снижение массы жировой ткани } \\
\text { 2. Увеличение мышечной массы } \\
\text { 3. Снижение концентрации глюкозы крови натощак }\end{array}$ & Проапоптотический эффект \\
\hline $\begin{array}{l}\text { Атеросклероз: } \\
\text { 1. Уменьшение толщины КИМ в эксперименте } \\
\text { 2. Уменьшение экспрессии VCAM-1 } \\
\end{array}$ & Гипертрофия и фиброз миокарда ЛЖ в эксперименте \\
\hline $\begin{array}{l}\text { Воспаление: } \\
\text { 1. Уменьшение концентраций IFN- } \gamma \text { и IL-6 }\end{array}$ & \\
\hline
\end{tabular}

апоптозе кардиомиоцитов, а также фиброзе миокарда крыс [37]. Экспериментальные данные согласуются с признаками гипертрофии миокарда у спортсменов, злоупотребляющих анаболическими/андрогенными стероидами, у которых существенно возрастает риск внезапной смерти.

Каруиоваскулярные эффекты тестостерона в экспериментальных моделях на животных

Когортные исследования выявили положительные корремяции между низкими уровнями эндогенного тестостерона и ишемической болезнью сердца, сахарным диабетом, инсулинорезистентностью [38]. В исследованиях случай-контроль пациенты с верифицированной ишемией миокарда имели значительно более низкие уровни тестостерона по сравнению с контрольной группой безпризнаков ишемической болезни сердца [12]. В метаанализе, включившем 12 наблюдательных исследований, была проанализирована связь между сывороточной концентрацией эндогенного тестостерона и общей смертностью (16 184 пациентов), а такжекардиоваскулярной смертностью (11 831 пациентов) [3]. Средний возраст пациентов составил 61 год, а средний период наблюдения составил 9,7 года. Относительный риск смерти от всех причин был выше у пациентов с низким содержанием эндогенного тестостерона [OP 1,35, 95\% $\triangle$ И 1,13-1,82, гетерогенность $\mathrm{I}^{2}=77,9 \%$, P<0,001], особенно у мужчин старше 60 лет (OP 1,54, $95 \%$ АИ 1,28-1,85) по сравнению с мужчинами моложе 60 лет (ОР 1,12, 95\% АИ 0,92-1,36). Тем не менее, снижение тестостерона может быть связано с наличием таких заболеваний, как сахарный диабет, ожирение, артериальная гипертония, курение табака, злоупотребление алкоголем, поэтому не исключается тот факт, что при проведении клинических исследований не были учтены данные потенциально искажающие факторы.

Каруиоваскулярные эффекты тестостерон-заместительной терапии

Большинство исследований, посвященных изучению эффекта ТЗТ на сердечно-сосудистые исходы имели следующие недостатки: 1) малый размер выборки, 2) включение здоровых асимптомных мужчин низкого кардиоваскулярного риска, 3) различные формы и дозы назначаемого тестостерона, 4) суррогатные конечные точки, 5) недостаточная статистическая мощность для сердечно-сосудистых событий.

\section{Ночное апноэ}

Результаты небольших наблюдательных исследований позволяют предположить, что даже физиологическое за- мещение тестостерона усиливает апноэ сна, состояние, к которому пожилые мужчины склонны вне зависимости от их уровня тестостерона. Тем не менее, в мета-анализе Calof et al. [9], опубликованном в 2005 г., частота апноэ сна не отличалась в группе ТЗТ и группе плацебо. В 2007 году Hanafy Н. опубликовал систематический обзор, в котором не было показано негативного влияния ТЗТ на риск возникновения и/или тяжесть апноэ сна [20]. Однако автор в своей статье подчеркнул низкий методологический уровень включенных в обзор исследований и сделал вывод о необходимости дальнейшего научного поиска.

В 2012 году были опубликованы результаты рандомизированного двойного слепого исследования, целью которого было изучения влияния ТЗТ на функцию внешнего дыхания у мужчин с ожирением и тяжелым синдромом обструктивного апноэ сна [25]. 67 участников исследования, находясь на низкокалорийной диете, были рандомизированы в группу инъекций тестостерона и группу плацебо. Сон и дыхание оценивались с помощью ночной полисомнографии в начале исследования, а также на 7 и 18 неделе. ТЗТ, по сравнению с плацебо, ухудшила кислородный индекс десатурации на 10,3 события/ч $(95 \%, \Delta$ И 0,8-19,8 событий/ч; $p=0,03)$ и индекс ночной гипоксемии (время сна с насыщением крови кислородом $<90 \%)$ на $6,1 \%\left(95 \% \Delta \Lambda_{1}, 1,5-10,6 ; p=0,01\right)$ через 7 недель. Тем не менее, через 18 недель в группе ТЗТ индекс десатурации $(4,5$ $14,4$ события/ч; $p=0,36)$, а также индекс ночной гипоксемии $(2,9-7,7 \%, p=0,23)$ достоверно не изменились по сравнению с группой плацебо. Таким образом, был продемонстрирован краткосрочный негативный эффект тестостерона на тяжесть ночного апноэ, который нивелировался к 18 неделе ТЗТ.

Те же авторы в 2012 году представили результаты исследования, оценивающего метаболические эффекты ТЗТ на той же когорте больных ожирением и тяжелым синдромом обструктивного апноэ сна [26]. Оказалось, что через 18 недель терапии ТЗТ привела к снижению инсулинорезистентности, снижению жировой массы и увеличению мышечной массы тела.

K сожалению, на сегодняшний день, не проведены рандомизированные исследования, изучающие долгосрочные эффекты ТЗТ на качество сна и выраженность ночного апноэ.

\section{Эритроцитоз}

ТЗТ может способствовать развитию эритроцитоза, особенно при использовании инъекционных форм тестостерона. 
В одном ретроспективном исследовании ТЗТ у 45 мужчин (средний возраст 71,8 лет), лечение тестостероном (200 мг внутримышечно каждые две недели) привело к достоверному увеличению риска эритроцитоза через 2 года наблюдения: у 11 пациентов (24\%) гематокрит вырос до 52\% и более (р $<0,001)$, что в ряде случаев потребовало кровопускания и во всех случаях отмены терапии. Несмотря на то, что самооценка либидо улучшилось в группе ТЗТ ( $<<0,0001)$, примерно треть пациентов прекратили лечение [19].

С 2008 года проведено лишь одно исследование, в котором изучалось влияние ТЗТ на уровень гемоглобина и гематокрита крови. Это исследование показало, что ТЗТ приводила к статистически значимому увеличению уровня гемоглобина крови $(0,86 \pm 0,31$ г/A1, $p=0,01)$. Механизм повышения уровня гемоглобина при ТЗТ до сих пор точно не установлен. Некоторое время стимуляцию эритропоэза при ТЗТ объясняли увеличением продукции эритропоэтина, однако эта теория была опровергнута. Тем не менее, в этом исследовании не доказан рост неблагоприятных сердечно-сосудистых событий на фоне повышения уровней гемоглобина и гематокрита крови в течение 36 месяцев наблюдения. В то же время, в исследовании Framingham, мужчины, уровень гематокрита которых превышал 46\%, имели большую общую и сердечнососудистую смертность [17].

На сегодняшний день не существует никаких других долгосрочных исследований, которые адекватно бы оценивали потенциальный риск эритроцитоза при ТЗТ. Практикующим врачам рекомендуется оценивать уровень гематокрита при старте ТЗТ и далее каждые 6 месяцев лечения. В случае, когда уровень гематокрита превышает 54\%, ТЗТ должна быть прекращена.

Венозные тромбоэмболии

Стандартная маркировка продукции тестостерона в США включает информацию о риске венозных тромбоэмболий (ВТЭ) вследствие эритроцитоза. Однако в настоящее время нет опубликованных исследований, в которых был бы продемонстрирован неблагоприятный эффект ТЗТ в отношении риска ВТЭ. Так в одном исследовании, 39 из 40 случаев ВТЭ у мужчин, принимающих тестостерон, были связаны с ранее не диагностированной тромбофилией. Это подчеркивает важность поиска случаев ВТЭ в личном и семейном анамнезе перед стартом ТЗТ. Рутинный скрининг на тромбофилию у мужчин, получающих ТЗТ, в настоящее время не рекомендуется $[5,18]$.

В исследовании, результаты которого были опубликованы J. Baillargeon et al. в 2015 году, не продемонстрировано увеличение риска ВТЭ на фоне ТЗТ [4]. В это исследование с дизайном случай-контроль были включены 30572 мужчин 40 лет и старше, которые были зачислены в одну из программ коммерческого страхования США в период с 1 января 2007 года до 31 декабря 2012 года. ТЗТ в течение 15 дней до даты события (ВТЭ) не повышала риск ВТЭ (ОР 0,90; 95\% АИ, 0,731,12). Важным является отсутствие повышения риска ВТЭ при любых путях введения тестостерона (подкожного [ОР 0,80, 95\% АИ 0,61-10,41], трансдермального [ОР 0,91; 95\% АИ 0,38-2,16] и внутримышечного [ОР 1,15; 95\% АИ 0,80-1,64]).

\section{Серgечно-сосудистая смертность}

Низкие концентрации эндогенного тестостерона связаны с повышенным риском развития сердечно-сосудистых заболеваний, в том числе ишемической болезни сердца и ишеми- ческого инсульта. Связан ли дефицит тестостерона напрямую с патогенезом сердечно-сосудистых заболеваний или же это маркер ранее существовавшего заболевания, лежащего в основе кардиоваскулярной патологии, остается неясным [12]. Аефицит тестостерона приводит к эндотелиальной дисфункции, прогрессии атеросклероза, инсулинорезистентности, поэтому теоретически способен повышать кардиоваскулярную смертность [48]. Создается впечатление, что ТЗТ, должна обладать мощным кардиопротективным действием, однако убедительных доказательств этого до сих пор не представлено.

Влияние тестостерона на субклинический атеросклероз у пожилых мужчин было изучено в исследовании ТЕААМ, опубликованном в 2015 году [6]. В этом исследовании 308 мужчин старше 60 лет с низким уровнем свободного тестостерона в сыворотке крови (<50 пг/мл [0,17 нмоль/л]) были рандомизированы в группу ТЗТ (1\% гель, 7,5 г) или плацебо. Период наблюдения составил 3 года. Средняя сывороточная концентрация тестостерона была выше в группе лечения по сравнению с группой плацебо (565 и 330 нг/Ал [19,6 и $11,4$ нмоль/ $]$, соответственно). При этом темпы изменения толщины интима-медиа (среднее различие с поправкой на возраст 0,0002 мм/год; 95\% $\Delta И,-0,003-0,003, \mathrm{p}=0,89$ ) и индекс коронарного кальция (скорректированное среднее различие, -10,8 Агэтстон единиц/год; 95\% $\Delta И,-45,7-24,2 ; p=0,54)$ существенно не отличались между группами.

Рандомизированное исследование ТЗТ (ТОМ) у пожилых мужчин (средний возраст 74 года) с ограничением подвижности и высокой распространенностью хронических заболеваний было прекращено досрочно, в связи с более высокой частотой сердечно-сосудистых событий в группе лечения тестостероном (ОР 2,4, p=0,05). Поскольку исследование было прекращено досрочно, а также вследствие того, что ни одно нежелательное явление не было преобладающим, трудно сделать обоснованные выводы о кардиоваскулярной безопасности ТЗТ у этой группы пациентов. Важно отметить и большую распространенность артериальной гипертензии, сахарного диабета, гиперлипидемии, ожирения и метаболического синдрома в группе ТЗТ [5].

Вторичный анализ исследования ТОМ показал, что через 6 месяцев терапии сывороточная концентрация свободного тестостерона была значительно выше у мужчин, перенесших сердечно-сосудистые события [95\% АИ, 10,6 (4,6-16,7) по сравнению с 5,2 (3,0-7,5) нг/Ал, p=0,05]. При использовании многовариантного логистического регрессионного анализа, повышение уровня свободного тестостерона крови на фоне ТЗТ, имело прямую связь с повышением риска сердечно-сосудистых катастроф.

Недавнее когортное исследование оценивало риск развития острого нефатального инфаркта миокарда в течение 90 дней после выдачи первого рецепта для ТЗТ. Авторы использовали базу данных здравоохранения США, охватывающую 55593 мужчин, которым назначалась ТЗТ [15]. В исследовании оценивалась также кардиоваскулярная безопасность ингибиторов фосфодиэстеразы 5 типа (силденафил или тадалафил) у 167279 мужчин. У мужчин в возрасте от 65 лет и старше в группе ТЗТ относительный риск был 2,19 (95\% АИ 1,27-3,77), в то время как в группе ингибиторов фосфодиэстеразы 5 типа OР составил 1,15 (95\% $\triangle$ И 0,83-1,59). Относительный риск нефатального инфаркта увеличивался с возрастом: от 0,95 (95\% АИ 0,54-1,67) Аля мужчин в возрасте до 55 лет и до 3,43 (95\% 
АИ 1,54-7,56) Аля мужчин в возрасте 75 лет и старше. ОАним из важнейших ограничений данного исследования является невозможность достоверно выяснить, является ли увеличение количества сердечно-сосудистых событий следствием базового дефицита тестостерона или же связано с ростом плазменных концентраций свободного тестостерона на фоне лечения.

В последние годы накопились доказательства большего кардиоваскулярного риска при использовании инъекционных форм тестостерона по сравнению с гелями. В ретроспективном анализе трех баз данных (более 544000 мужчин, получающих ТЗТ), применение инъекционных препаратов тестостерона было связано с большим риском развития инфаркта миокарда (ОР 1,30, 95\% АИ 1,18-1,45) и инсульта (ОР 1,21, 95\% АИ 1,10$1,32)$, но не ВТЭ (ОР 0,92), по сравнению с гелем тестостерона. При этом абсолютный риск инфаркта миокарда и инсульта был низким (1 и 5 дополнительных событий/1000 пациентолет Аля мужчин до 60 лет и старше 65 лет, соответственно). Аанный факт можно объяснить прерывистым созданием в крови супрафизиологических концентраций тестостерона при использовании инъекционных форм [30].

Проведенные мета-анализы показывают очень противоречивые результаты и не позволяют сделать окончательных выводов: 3 мета-анализа не выявили неблагоприятных эффектов ТЗТ в отношении риска сердечно-сосудистых событий, 2 метаанализа свидетельствуют об обратном. Интересной является найденная зависимость между результатами исследований и источником финансирования. В мета-анализе L. Xu и et al., опубликованном в 2013 году [43], эффективность ТЗТ прямо коррелировала с источником финансирования исследований $(p=0,03)$, но не с исходным уровнем тестостерона $(p=0,70)$. В ходе испытаний, не финансируемых фармацевтической промышленностью, риск сердечно-сосудистых событий в группе ТЗТ был выше (ОР 2,06, 95\% АИ 1,34-3,17), чем в спонсируемых исследованиях (ОР 0,89, 95\% АИ 0,50-1,60).

Резюмируя, необходимо отметить, что ТЗТ существенно улучшит качество жизни пациентов с симптомами андрогенного дефицита в результате заболевания семенников, гипофиза или гипоталамуса. Тем не менее, убедительных доказательств кардиоваскулярной безопасности ТЗТ до настоящего времени не получено. Справедливо отметить тот факт, что не продемонстрировано и доказанное повышение кардиоваскулярной смертности на фоне лечения. Несмотря на это, в последние десятилетия отмечается впечатляющее увеличение процентного содержания рецептов на препараты тестостерона с 0,81\% в 2001 году до 2,91\% в 2011 году среди мужчин старше 40 лет. За тот же период в Великобритании количество выписываемых рецептов на препараты тестостерона выросло на 90\%, хотя распространенность дефицита тестостерона осталась неизменной [44]. Это послужило сигналом для FDA в 2014 году выступить с заявлением, в котором излагаются опасения в отношении сердечно-сосудистой безопасности ТЗТ. С этого времени производителям препаратов тестостерона рекомендовано Аобавлять информацию о возможном увеличении риска инфаркта миокарда и ишемического инсульта у пациентов, принимающих тестостерон [45]. Очевидно, что пока не будут проведены крупные рандомизированные двойные слепые исследования с достаточным количеством событий в группах сравнения, неправомочно делать выводы о безопасности ТЗТ у пациентов с возрастным дефицитом тестостерона.

\section{Аитература}

1. Alexandersen P, Haarbo J, Byrjalsen I, Lawaetz $H_{1}$ Christiansen C. Natural androgens inhibit male atherosclerosis: a study in castrated, cholesterol-fed rabbits. Circulation Research. 1999;84:813-9.

2. Ammar EM, Said SA, Hassan MS. Enhanced vasoconstriction and reduced vasorelaxation induced by testosterone and nandrolone in hypercholesterolemic rabbits. Pharmacological Research. 2004;50:253 - 9. DOI:10.1016/j.phrs.2004.03.010.

3. Araujo AB, Dixon JM, Suarez EA, Murad MH, Guey LT, Wittert GA. Endogenous testosterone and mortality in men: a systematic review and meta-analysis. The Journal of Clinical Endocrinology and Metabolism. 2011;96:3007-19. DOI: 10.1210/jc.2011-1137.

4. Baillargeon J, Urban RJ, Morgentaler A, Glueck CJ, Baillargeon G, Sharma G, Kuo YF. Risk of Venous Thromboembolism in Men Receiving Testosterone Therapy. Mayo Clinic Proceedings. 2015;90(8):1038-45. DOI: 10.1016/j.mayocp.2015.05.012.

5. Basaria $S$, Coviello AD, Travison TG, Storer TW, Farwell WR, Jette AM, Eder R, Tennstedt $S$, Ulloor J, Zhang A, Choong K, Lakshman KM, Mazer NA, Miciek R, Krasnoff J, Elmi A, Knapp PE, Brooks B, Appleman E, Aggarwal S, Bhasin G, Hede-Brierley L, Bhatia A, Collins L, LeBrasseur N, Fiore LD, Bhasin S. Adverse events associated with testosterone administration. The New England Journal of Medicine. 2010; 363:109. DOI: 10.1056/NEJMoa1000485.

6. Basaria $\mathrm{S}$, Harman SM, Travison TG, Hodis $\mathrm{H}_{1}$ Tsitouras $\mathrm{P}$, Budoff M, Pencina KM, Vita J, Dzekov C, Mazer NA, Coviello AD, Knapp PE, Hally K, Pinjic E, Yan M, Storer TW, Bhasin S. Effects of Testosterone Administration for 3 Years on Subclinical Atherosclerosis Progression in Older Men With Low or Low-Normal Testosterone Levels: A Randomized Clinical Trial. JAMA. 2015;314:570. DOI: 10.1001/jama.2015.8881.

7. Brand JS, van der Tweel I, Grobbee DE, Emmelot-Vonk MH, van der Schouw YT. Testosterone, sex hormone-binding globulin and the metabolic syndrome: a systematic review and meta-analysis of observational studies. International Journal of Epidemiology. 2011;40:189 207. DOI: $10.1093 /$ ije/dyq158.

8. Bushnell C, McCullough LD, Awad IA, Chireau MV, Fedder WN, Furie KL, Howard VJ, Lichtman JH, Lisabeth LD, Piña IL, Reeves MJ, Rexrode KM, Saposnik G, Singh V, Towfighi A, Vaccarino V, Walters MR. Guidelines for the prevention of stroke in women: a statement for healthcare professionals from the American Heart Association/American Stroke Association. Stroke. 2014;45:1545. DOI: 10.1161/01.str.0000442009.06663.48.

9. Calof OM, Singh AB, Lee ML, Kenny AM, Urban RJ, Tenover JL, Bhasin S. Adverse events associated with testosterone replacement in middle-aged and older men: a meta-analysis of randomized, placebocontrolled trials. The Journals of Gerontology. Series A, Biological Sciences and Medical Sciences.2005;60:1451 - 7.

10. Cavasin MA, Tao ZY, Yu AL, Yang XP. Testosterone enhances early cardiac remodeling after myocardial infarction, causing rupture and degrading cardiac function. American Journal of Physiology. Heart and Circulatory Physiology. 2006;290:2043 - 50. DOI: 10.1152/ ajpheart.01121.2005.

11. Ceballos G, Figueroa L, Rubio I, Gallo G, Garcia A, Martinez A, Yanez R, Perez J, Morato T, Chamorro G. Acute and nongenomic effects of testosterone on isolated and perfused rat heart. Journal of Cardiovascular Pharmacology. 1999;33:691 - 7.

12. Corona G, Rastrelli G, Monami M et al. Hypogonadism as a risk factor for cardiovascular mortality in men: a meta-analytic study. European Journal of Endocrinology. 2011;165(5):687-701. DOI: 10.1530/EJE-11-0447. 
13. Crisostomo PR, Wang M, Wairiuko GM, Morrell ED, Meldrum DR. Brief exposure to exogenous testosterone increases death signaling and adversely affects myocardial function after ischemia. American Journal of Physiology. Regulatory, Integrative and Comparative Physiology. 2006;290:1168 - 74. DOI: 10.1152/ajpregu.00833.2005.

14. Feldman HA, Longcope $C$, Derby $C A$, Johannes $C B$, Araujo $A B$, Coviello AD, Bremner WJ, McKinlay JB. Age trends in the level of serum testosterone and other hormones in middle-aged men: longitudinal results from the Massachusetts male aging study. The Journal of Clinical Endocrinology and Metabolism. 2002;87:589-98. DOI: 10.1210/jcem.87.2.8201.

15. Finkle WD, Greenland S, Ridgeway GK, Adams JL, Frasco MA, Cook MB, Fraumeni JF Jr, Hoover RN. Increased risk of non-fatal myocardial infarction following testosterone therapy prescription in men. PLOS One. 2014;9(1):e85805. DOI: 10.1371/journal.pone.0085805.

16. Flake NM, Hermanstyne TO, Gold MS. Testosterone and estrogen have opposing actions on inflammation-induced plasma extravasation in the rat temporomandibular joint. American Journal of Physiology. Regulatory, Integrative and Comparative Physiology. 2006;2291(2):R343-8. DOI: 10.1152/ajpregu.00835.2005.

17. Gagnon DR, Zhang TJ, Brand FN, Kannel WB. Hematocrit and the risk of cardiovascular disease--the Framingham study: a 34-year follow-up. American Heart Journal. 1994;127(3):674-82.

18. Glueck CJ, Wang P. Testosterone therapy, thrombosis, thrombophilia, cardiovascular events. Metabolism 2014;63(8):989-94. DOI: 10.1016/j.metabol.2014.05.005.

19. Hajjar RR, Kaiser FE, Morley JE. Outcomes of long-term testosterone replacement in older hypogonadal males: a retrospective analysis. The Journal of Clinical Endocrinology and Metabolism. 1997;82(11):3793-6. DOI: 10.1210/jcem.82.11.4387.

20. Hanafy HM. Testosterone therapy and obstructive sleep apnea: is there a real connection? The Journal of Sexual Medicine. 2007;4(5):1241-6. DOI: 10.1210/jcem.82.11.4387.

21. Hanke H, Lenz C, Hess B, Spindler KD, Weidemann W. Effect of testosterone on plaque development and androgen receptor expression in the arterial vessel wall. Circulation. 2001;103(10):1382 - 5.

22. Harman SM, Metter EJ, Tobin JD, Pearson J, Blackman MR. Longitudinal effects of aging on serum total and free testosterone levels in healthy men. Baltimore Longitudinal Study of Aging. The Journal of Clinical Endocrinology and Metabolism. 2001;86(2):724-31. DOI: $10.1210 /$ jcem.86.2.7219.

23. Herring MJ, Oskui PM, Hale SL, Kloner RA. Testosterone and the cardiovascular system: a comprehensive review of the basic science literature. Journal of the American Heart Association. 2013;2(4):e000271. DOI: 10.1161/JAHA.113.000271.

24. Hofbauer LC, Ten RM, Khosla S. The anti-androgen hydroxyflutamide and androgens inhibit interleukin-6 production by an androgen-responsive human osteoblastic cell line. Journal of Bone and Mineral Research. 1999;14(8):1330-7. DOI: 10.1359/ jbmr.1999.14.8.1330.

25. Hoyos CM, Killick R, Yee BJ, Grunstein RR, Liu PY. Effects of testosterone therapy on sleep and breathing in obese men with severe obstructive sleep apnoea: a randomized placebo-controlled trial. Clinical Endocrinology (Oxford). 201277(4):599-607. DOI: 10.1111/j.1365-2265.2012.04413.x.

26. Hoyos CM, Yee BJ, Phillips CL, Machan EA, Grunstein RR, Liu PY. Body compositional and cardiometabolic effects of testosterone therapy in obese men with severe obstructive sleep apnoea: a randomised placebo-controlled trial. European Journal of Endocrinology. 2012;167(4):531-41. doi: 10.1530/EJE-12-0525.
27. Прогноз ожидаемой продолжительности жизни в Российской Федерации к 2030 году[Интернет]. Федеральная служба государственной статистики. Аоступно: http://www.gks.ru/wps/ wcm/connect/rosstat_main/rosstat/ru/statistics/population/ demography/\#

28. Kupelian V, Chiu GR, Araujo AB, Williams RE, Clark RV, McKinlay JB. Association of sex hormones and C-reactive protein levels in men. Clinical Endocrinology (Oxford). 2010;72(4):527-33. DOI: 10.1111/j.1365-2265.2009.03713.x.

29. Lakshman KM, Bhasin S, Araujo AB. Sex hormone-binding globulin as an independent predictor of incident type 2 diabetes mellitus in men. The Journals of Gerontology. Series A, Biological Sciences and Medical Sciences. 2010;65(5):503-9. DOI: 10.1093/ gerona/glq002.

30. Layton JB, Meier CR, Sharpless JL, Stürmer T, Jick SS, Brookhart MA. Comparative Safety of Testosterone Dosage Forms. JAMA Internal Medicine. 2015;175(7):1187-96. doi: 10.1001/ jamainternmed.2015.1573.

31. Li GR, Feng J, Wang Z, Fermini B, Nattel S. Adrenergic modulation of ultrarapid delayed rectifier $\mathrm{K}+$ current in human atrial myocytes. Circulation Research. 1996;78(5):903-15.

32. Liu A, Gao L, Kang S, Liu Y, Xu C, Sun H, Li D, Yan C. Testosterone enhances estradiol's cardioprotection in ovariectomized rats. The Journal of Endocrinology. 2012;212(1):61-9. DOI: 10.1530/ JOE-11-0181.

33. Liu J, Tsang S, Wong TM. Testosterone is required for delayed cardioprotection and enhanced heat shock protein 70 expression induced by preconditioning. Endocrinology. 2006;147(10):4569-77. DOI: 10.1210/en.2006-0297.

34. McCrohon JA, Jessup W, Handelsman DJ, Celermajer DS. Androgen exposure increases human monocyte adhesion to vascular endothelium and endothelial cell expression of vascular cell adhesion molecule-1. Circulation. 1999;99(17):2317-22.

35. Nathan L, Shi W, Dinh H, Mukherjee TK, Wang X, Lusis AJ, Chaudhuri G. Testosterone inhibits early atherogenesis by conversion to estradiol: critical role of aromatase. Proceedings of the National Academy of Sciences of the United States of America. 2001;98(6):3589-93.

36. Ng MK, Quinn CM, McCrohon JA, Nakhla S, Jessup W, Handelsman DJ, Celermajer DS, Death AK. Androgens up-regulate atherosclerosis-related genes in macrophages from males but not females: molecular insights into gender differences in atherosclerosis. Journal of the American College of Cardiology. 2003;42(7):1306-13.

37. Papamitsou T, Barlagiannis D, Papaliagkas V, Kotanidou E, Dermentzopoulou-Theodoridou M. Testosterone-induced hypertrophy, fibrosis and apoptosis of cardiac cells-an ultrastructural and immunohistochemical study. Medical Science Monitor. 2011;17(9):BR266-73.

38. Srinath R, Hill Golden $\mathrm{S}$, Carson KA, Dobs A. Endogenous testosterone and its relationship to preclinical and clinical measures of cardiovascular disease in the atherosclerosis risk in communities study. The Journal of Clinical Endocrinology and Metabolism. 2015;100(4):1602-8. DOI: 10.1210/jc.2014-3934.

39. Stellato RK, FeldmanHA, Hamdy O, Horton ES, McKinlay JB. Testosterone, sex hormone-binding globulin, and the development of type 2 diabetes in middle-aged men: prospective results from the Massachusetts male aging study. Diabetes Care. 2000;23(4):490-4.

40. Stuenkel CA, Davis SR, Gompel A, Lumsden MA, Murad MH, Pinkerton JV, Santen RJ. Treatment of Symptoms of the Menopause: 
An Endocrine Society Clinical Practice Guideline. The Journal of Clinical Endocrinology and Metabolism.2015;100(11):3975-4011. DOI: 10.1210/jc.2015-2236.

41. Tsang S, Wu S, Liu J, Wong TM. Testosterone protects rat hearts against ischaemic insults by enhancing the effects of alpha(1)-adrenoceptor stim1.ulation. British Journal of Pharmacology. 2008;153(4):693-709. DOI: 10.1038/sj.bjp.0707624.

42. Wu FC, Tajar A, Beynon JM, Pye SR, Silman AJ, Finn JD, O'Neill TW, Bartfai G, Casanueva FF, Forti G, Giwercman A, Han TS, Kula K, Lean ME, Pendleton N, Punab M, Boonen S, Vanderschueren D, Labrie F, Huhtaniemi IT. Identification of late-onset hypogonadism in middle-aged and elderly men. The New England Journal of Medicine. 2010;363(2):123-35. DOI: 10.1056/NEJMoa0911101.

43. Xu L, Freeman G, Cowling B, Schooling C. Testosterone therapy and cardiovascular events among men: a systematic review and meta-analysis of placebo-controlled randomized trials. BMC Medicine. 2013;11:108. DOI: 10.1186/1741-7015-11-108.

44. Garnick MB. Testosterone replacement therapy faces FDA scrutiny. JAMA. 2015;313(6):563-4. DOI: 10.1001/jama.2014.17334.

45. FDA Drug Safety Communication: FDA cautions about using testosterone products for low testosterone due to aging; requires labeling change to inform of possible increased risk of heart attack and stroke with use. The Journal of Urology. 2015;194(3):759-60. DOI: 10.1016/j.juro.2015.06.058.

\section{References}

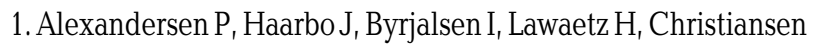
C. Natural androgens inhibit male atherosclerosis: a study in castrated, cholesterol-fed rabbits. Circulation Research. 1999;84:813-9.

2. Ammar EM, Said SA, Hassan MS. Enhanced vasoconstriction and reduced vasorelaxation induced by testosterone and nandrolone in hypercholesterolemic rabbits. Pharmacol Res. 2004;50:253259. DOI:10.1016/j.phrs.2004.03.010.

3. Araujo AB, Dixon JM, Suarez EA, Murad MH, Guey LT, Wittert GA. Endogenous testosterone and mortality in men: a systematic review and meta-analysis. The Journal of Clinical Endocrinology and Metabolism. 2011;96:3007-19. DOI: 10.1210/jc.2011-1137.

4. Baillargeon J, Urban RJ, Morgentaler A, Glueck CJ, Baillargeon G, Sharma G, Kuo YF. Risk of Venous Thromboembolism in Men Receiving Testosterone Therapy. Mayo Clinic Proceedings.2015;90(8):1038-1045. DOI: 10.1016/j.mayocp.2015.05.012.5.

5. Basaria S, Coviello AD, Travison TG, Storer TW, Farwell WR, Jette AM, Eder R, Tennstedt S, Ulloor J, Zhang A, Choong K, Lakshman KM, Mazer NA, Miciek R, Krasnoff J, Elmi A, Knapp PE, Brooks B, Appleman E, Aggarwal S, Bhasin G, Hede-Brierley L, Bhatia A, Collins L, LeBrasseur N, Fiore LD, Bhasin S. Adverse events associated with testosterone administration. The New England Journal of Medicine. 2010; 363:109. DOI: 10.1056/NEJMoa1000485.

6. Basaria $S_{1}$ Harman SM, Travison TG, Hodis $H_{1}$ Tsitouras $P_{\text {, }}$ Budoff M, Pencina KM, Vita J, Dzekov C, Mazer NA, Coviello AD, Knapp PE, Hally K, Pinjic E, Yan M, Storer TW, Bhasin S. Effects of Testosterone Administration for 3 Years on Subclinical Atherosclerosis Progression in Older Men With Low or Low-Normal Testosterone Levels: A Randomized Clinical Trial. JAMA. 2015;314:570. DOI: 10.1001/jama.2015.8881.

7. Brand JS, van der Tweel I, Grobbee DE, Emmelot-Vonk MH, van der Schouw YT. Testosterone, sex hormone-binding globulin and the metabolic syndrome: a systematic review and meta-analysis of observational studies. International Journal of Epidemiology. 2011;40:189 207. DOI: 10.1093/ije/dyq158.
8. Bushnell C, McCullough LD, Awad IA, Chireau MV, Fedder WN, Furie KL, Howard VJ, Lichtman JH, Lisabeth LD, Piña IL, Reeves MJ, Rexrode KM, Saposnik G, Singh V, Towfighi A, Vaccarino V, Walters MR. Guidelines for the prevention of stroke in women: a statement for healthcare professionals from the American Heart Association/American Stroke Association. Stroke. 2014;45:1545. DOI: 10.1161/01.str.0000442009.06663.48.

9. Calof OM, Singh AB, Lee ML, Kenny AM, Urban RJ, Tenover JL, Bhasin S. Adverse events associated with testosterone replacement in middle-aged and older men: a meta-analysis of randomized, placebocontrolled trials. The Journals of Gerontology. Series A, Biological Sciences and Medical Sciences.2005;60:1451 - 7.

10. Cavasin MA, Tao ZY, Yu AL, Yang XP. Testosterone enhances early cardiac remodeling after myocardial infarction, causing rupture and degrading cardiac function. American Journal of Physiology. Heart and Circulatory Physiology. 2006;290:2043 - 50. DOI: 10.1152/ ajpheart.01121.2005.

11. Ceballos G, Figueroa L, Rubio I, Gallo G, Garcia A, Martinez A, Yanez R, Perez J, Morato T, Chamorro G. Acute and nongenomic effects of testosterone on isolated and perfused rat heart. Journal of Cardiovascular Pharmacology. 1999;33: 691 - 97.

12. Corona G, Rastrelli G, Monami M et al. Hypogonadism as a risk factor for cardiovascular mortality in men: a meta-analytic study. European Journal of Endocrinology. 2011;165(5):687-701. DOI: 10.1530/EJE-11-0447.

13. Crisostomo PR, Wang M, Wairiuko GM, Morrell ED, Meldrum DR. Brief exposure to exogenous testosterone increases death signaling and adversely affects myocardial function after ischemia. American Journal of Physiology. Regulatory, Integrative and Comparative Physiology. 2006;290:1168 - 74. DOI: 10.1152/ajpregu.00833.2005.

14. Feldman HA, Longcope $C$, Derby CA, Johannes CB, Araujo $A B$, Coviello AD, Bremner WJ, McKinlay JB. Age trends in the level of serum testosterone and other hormones in middle-aged men: longitudinal results from the Massachusetts male aging study. The Journal of Clinical Endocrinology and Metabolism. 2002;87:589-98. DOI: 10.1210/jcem.87.2.8201.

15. Finkle WD, Greenland S, Ridgeway GK, Adams JL, Frasco MA, Cook MB, Fraumeni JF Jr, Hoover RN. Increased risk of nonfatal myocardial infarction following testosterone therapy prescription in men. PLOS One. 2014;9(1):e85805. DOI: 10.1371/journal. pone.0085805.

16. Flake NM, Hermanstyne TO, Gold MS. Testosterone and estrogen have opposing actions on inflammation-induced plasma extravasation in the rat temporomandibular joint. American Journal of Physiology. Regulatory, Integrative and Comparative Physiology. 2006;2291(2):R343-8. DOI: 10.1152/ajpregu.00835.2005.

17. Gagnon DR, Zhang TJ, Brand FN, Kannel WB. Hematocrit and the risk of cardiovascular disease--the Framingham study: a 34-year follow-up. American Heart Journal. 1994;127(3):674-82.

18. Glueck CJ, Wang P. Testosterone therapy, thrombosis, thrombophilia, cardiovascular events. Metabolism 2014;63(8):989-94. DOI: 10.1016/j.metabol.2014.05.005.

19. Hajjar RR, Kaiser FE, Morley JE. Outcomes of long-term testosterone replacement in older hypogonadal males: a retrospective analysis. The Journal of Clinical Endocrinology and Metabolism. 1997;82(11):3793-6. DOI: 10.1210/jcem.82.11.4387.

20. Hanafy HM. Testosterone therapy and obstructive sleep apnea: is there a real connection? The Journal of Sexual Medicine. 2007;4(5):1241-6. DOI: 10.1210/jcem.82.11.4387.

21. Hanke H, Lenz C, Hess B, Spindler KD, Weidemann W. Effect 
of testosterone on plaque development and androgen receptor expression in the arterial vessel wall. Circulation. 2001;103(10):1382 - 5 .

22. Harman SM, Metter EJ, Tobin JD, Pearson J, Blackman MR. Longitudinal effects of aging on serum total and free testosterone levels in healthy men. Baltimore Longitudinal Study of Aging. The Journal of Clinical Endocrinology and Metabolism. 2001;86(2):724-31. DOI: $10.1210 /$ jcem.86.2.7219.

23. Herring MJ, Oskui PM, Hale SL, Kloner RA. Testosterone and the cardiovascular system: a comprehensive review of the basic science literature. Journal of the American Heart Association. 2013;2(4):e000271. DOI: 10.1161/JAHA.113.000271.

24. Hofbauer LC, Ten RM, Khosla S. The anti-androgen hydroxyflutamide and androgens inhibit interleukin-6 production by an androgen-responsive human osteoblastic cell line. Journal of Bone and Mineral Research. 1999;14(8):1330-7. DOI: 10.1359/ jbmr.1999.14.8.1330.

25. Hoyos CM, Killick R, Yee BJ, Grunstein RR, Liu PY. Effects of testosterone therapy on sleep and breathing in obese men with severe obstructive sleep apnoea: a randomized placebo-controlled trial. Clin Endocrinol (Oxf). 2012a; 77:599-607.

26. Hoyos CM, Yee BJ, Phillips CL, Machan EA, Grunstein RR, Liu PY. Body compositional and cardiometabolic effects of testosterone therapy in obese men with severe obstructive sleep apnoea: a randomised placebo-controlled trial. European Journal of Endocrinology. 2012;167(4):531-41. doi: 10.1530/EJE-12-0525.

27. Forecast of life expectancy in the Russian Federation by 2030 [Internet]. Federal Service of State Statistics. Available from: http:// www.gks.ru/wps/wcm/connect/rosstat_main/rosstat/ru/statistics/ population/demography/\#

28. Kupelian V, Chiu GR, Araujo AB, Williams RE, Clark RV, McKinlay JB. Association of sex hormones and C-reactive protein levels in men. Clinical Endocrinology (Oxford). 2010;72(4):527-33. DOI: 10.1111/j.1365-2265.2009.03713.x.

29. Lakshman KM, Bhasin S, Araujo AB. Sex hormone-binding globulin as an independent predictor of incident type 2 diabetes mellitus in men. The Journals of Gerontology. Series A, Biological Sciences and Medical Sciences. 2010;65(5):503-9. DOI: 10.1093/gerona/glq002.

30. Layton JB, Meier CR, Sharpless JL, Stürmer T, Jick SS, Brookhart MA. Comparative Safety of Testosterone Dosage Forms. JAMA Internal Medicine. 2015;175(7):1187-96. doi: 10.1001/ jamainternmed.2015.1573.

31. Li GR, Feng J, Wang Z, Fermini B, Nattel S. Adrenergic modulation of ultrarapid delayed rectifier $\mathrm{K}+$ current in human atrial myocytes. Circulation Research. 1996;78(5):903-15.

32. Liu A, Gao L, Kang S, Liu Y, Xu C, Sun H, Li D, Yan C. Testosterone enhances estradiol's cardioprotection in ovariectomized rats. The Journal of Endocrinology. 2012;212(1):61-9. DOI: 10.1530/JOE-11-0181.

33. Liu J, Tsang S, Wong TM. Testosterone is required for delayed cardioprotection and enhanced heat shock protein 70 expression induced by preconditioning. Endocrinology. 2006;147(10):4569-77. DOI: 10.1210/en.2006-0297.

34. McCrohon JA, Jessup W, Handelsman DJ, Celermajer DS. Androgen exposure increases human monocyte adhesion to vascular endothelium and endothelial cell expression of vascular cell adhesion molecule-1. Circulation. 1999;99(17):2317-22.

35. Nathan L, Shi W, Dinh H, Mukherjee TK, Wang X, Lusis AJ, Chaudhuri G. Testosterone inhibits early atherogenesis by conversion to estradiol: critical role of aromatase. Proceedings of the National Academy of Sciences of the United States of America. 2001;98(6):3589-93.
36. Ng MK, Quinn CM, McCrohon JA, Nakhla S, Jessup W, Handelsman DJ, Celermajer DS, Death AK. Androgens up-regulate atherosclerosis-related genes in macrophages from males but not females: molecular insights into gender differences in atherosclerosis. Journal of the American College of Cardiology. 2003;42(7):1306-13.

37. Papamitsou T, Barlagiannis D, Papaliagkas V, Kotanidou E, Dermentzopoulou-Theodoridou M. Testosterone-induced hypertrophy, fibrosis and apoptosis of cardiac cells-an ultrastructural and immunohistochemical study. Medical Science Monitor. 2011;17(9):BR266-73.

38. Srinath R, Hill Golden S, Carson KA, Dobs A. Endogenous testosterone and its relationship to preclinical and clinical measures of cardiovascular disease in the atherosclerosis risk in communities study. The Journal of Clinical Endocrinology and Metabolism. 2015;100(4):1602-8. DOI: 10.1210/jc.2014-3934.

39. Stellato RK, FeldmanHA, Hamdy O, Horton ES, McKinlay JB. Testosterone, sex hormone-binding globulin, and the development of type 2 diabetes in middle-aged men: prospective results from the Massachusetts male aging study. Diabetes Care. 2000;23(4):490-4.

40. Stuenkel CA, Davis SR, Gompel A, Lumsden MA, Murad MH, Pinkerton JV, Santen RJ. Treatment of Symptoms of the Menopause: An Endocrine Society Clinical Practice Guideline. The Journal of Clinical Endocrinology and Metabolism.2015;100(11):3975-4011. DOI: 10.1210/jc.2015-2236.

41. Tsang S, Wu S, Liu J, Wong TM. Testosterone protects rat hearts against ischaemic insults by enhancing the effects of alpha(1)-adrenoceptor stimulation. British Journal of Pharmacology. 2008;153(4):693-709. DOI: 10.1038/sj.bjp.0707624.

42. Wu FC, Tajar A, Beynon JM, Pye SR, Silman AJ, Finn JD, O'Neill TW, Bartfai G, Casanueva FF, Forti G, Giwercman A, Han TS, Kula K, Lean ME, Pendleton N, Punab M, Boonen S, Vanderschueren D, Labrie F, Huhtaniemi IT. Identification of late-onset hypogonadism in middle-aged and elderly men. The New England Journal of Medicine. 2010;363(2):123-35. DOI: 10.1056/NEJMoa0911101.

43. Xu L, Freeman G, Cowling B, Schooling C. Testosterone therapy and cardiovascular events among men: a systematic review and meta-analysis of placebo-controlled randomized trials. $B M C$ Medicine. 2013;11:108. DOI: 10.1186/1741-7015-11-108.

44. Garnick MB. Testosterone replacement therapy faces FDA scrutiny. JAMA. 2015;313(6):563-4. DOI: 10.1001/jama.2014.17334.

45. FDA Drug Safety Communication: FDA cautions about using testosterone products for low testosterone due to aging; requires labeling change to inform of possible increased risk of heart attack and stroke with use. The Journal of Urology. 2015;194(3):759-60. DOI: 10.1016/j.juro.2015.06.058.

\section{Сведения об авторах}

Беряников Сергей Валерьевич, Европейский меgицинский центр; аgрес: Российская Феgерация, 129090, г. Москва, ул. Щепкина, g. 35; тел.: +7(915)2920371; e-mail: sergey.v.berdnikov@gmail.com

Беруникова Ольга Александровна, Орловская областная кииническая больница; аgрес: Российская Феgерация, 302028, г. Орел, ул. Побеgы бульвар, g. 10; тел.: +7(980)3683105; e-mail: berline@list.ru

\section{Information about the authors}

Berdnikov Sergey Valerievich, European medical center, Cardiologist; Address: 35, Schepkina Str., Moscow, Russian Federation 129090; Phone: +7(915)2920371; e-mail: sergey.v.berdnikov@gmail.com

Berdnikova Olga Aleksandrovna, General Practitioner, Nephrologist, Oryol Regional Hospital; Address: 10, Pobedy Bulvar Str., Oryol, Russian Federation 302028; Phone:+7(980)3683105; e-mail: berline@list.ru 